\title{
ON GLOBAL INSTABILITY OF SOLUTIONS TO HYPERBOLIC EQUATIONS WITH NON-LIPSCHITZ NONLINEARITY
}

\author{
Y.Sh. IL'YASOV, E.E. KHOLODNOV
}

\begin{abstract}
In a bounded domain $\Omega \subset \mathbb{R}^{n}$, we consider the following hyperbolic equation

$$
\left\{\begin{array}{l}
v_{t t}=\Delta_{p} v+\lambda|v|^{p-2} v-|v|^{\alpha-2} v, \quad x \in \Omega, \\
\left.v\right|_{\partial \Omega}=0 .
\end{array}\right.
$$

We assume that $1<\alpha<p<+\infty$; this implies that the nonlinearity in the right hand side of the equation is of a non-Lipschitz type. As a rule, this type of nonlinearity prevent us from applying standard methods from the theory of nonlinear differential equations. An additional difficulty arises due to the presence of the $p$-Laplacian $\Delta_{p}(\cdot):=\operatorname{div}\left(|\nabla(\cdot)|^{p-2} \nabla(\cdot)\right)$ in the equation. In the first result, the theorem on the existence of the so-called stationary ground state of the equation is proved. The proof of this result is based on the Nehari manifold method. In the main result of the paper we state that each stationary ground state is unstable globally in time. The proof is based on the development of an approach by Payne and Sattinger introduced for studying the stability of solutions to hyperbolic equations.
\end{abstract}

Keywords: stability of solutions, nonlinear hyperbolic equations, Nehari manifold method, $p$-Laplacian

Mathematics Subject Classification: 35J61, 35J92, 35J50

\section{INTRODUCTION}

We consider the Dirichlet boundary value problem

$$
\left\{\begin{aligned}
-\Delta_{p} u & =\lambda|u|^{p-2} u-|u|^{\alpha-2} u, \quad x \in \Omega, \\
\left.u\right|_{\partial \Omega} & =0,
\end{aligned}\right.
$$

where $\Omega$ is a bounded domain in $\mathbb{R}^{n}$ with a smooth boundary $\partial \Omega, n \geqslant 1, \lambda \in \mathbb{R}$. The symbol $\Delta_{p}(\cdot):=\operatorname{div}\left(|\nabla(\cdot)|^{p-2} \nabla(\cdot)\right)$ stands for the $p$-Laplacian, $1<p<+\infty$. We suppose that $\alpha \in(1, p)$, that is, the nonlinearity in the right hand side is of a non-Lipschitz type. A special interest to such problem related to the fact that they can possess solutions compactly supported in $\Omega$ (see, for instance, [1], [2], [3], [5], [16] and the review of the references therein). However, despite there are rather many works devoted to such solutions, their stability for the corresponding non-stationary problems: parabolic and hyperbolic equations, Schrödinger equations, etc., was studied to a lesser extent. Here the main obstacle is that the presence of the non-Lipschitz nonlinearity in the right hand side of (B.1) makes troubles in applying the methods based on studying the corresponding linearized equations. Another difficulty is that

Y.Sh. IL'yasov, E.E. KhOLODNOV, On GLOBAL INSTABILITy OF SOLUTIONS TO HyPERBOLIC EQUATIONS WITH NON-LIPSCHITZ NONLINEARITY.

(C)IL'Yasov Y.Sh., Kholodnov E.E. 2017.

Submitted August 28, 2017. 
the most known results on existence of solutions compactly supported in with $\Omega$ are usually nonconstructive and are of an abstract nature. This prevents one from further studying detailed properties like, for example, stability for such solutions.

In works [4], [5], [8], [10], for equations with non-Lipschitz nonlinearities, there were found solutions compactly supported in $\Omega$ and in addition, these solutions were ground states. This additional property turned out to allow one to obtain certain stability results for such solutions. In particular, in work [4] there were obtained stability results for solutions to (B.1) (as $p=2)$, which were of ground state kind compactly supported in $\Omega$; the results were obtained for the corresponding parabolic problem.

The aim of the present work is to study the stability of the stationary solutions to the hyperbolic equation

$$
\left\{\begin{array}{l}
v_{t t}=\Delta_{p} v+\lambda|v|^{p-2} v-|v|^{\alpha-2} v, \quad x \in \Omega, \\
\left.v\right|_{t=0}=v_{0} \\
\left.v_{t}\right|_{t=0}=v_{1} \\
\left.v\right|_{\partial \Omega}=0
\end{array}\right.
$$

In order to do this, we first prove the existence of stationary ground state kind solutions to equation (B.2). The proof of this result is based on employing the Nehari manifold method. Such approach allows us to obtain some additional qualitative geometric properties of these solutions required for further purposes. In the main result we prove that the stationary ground state kind solutions to hyperbolic problem $(\overline{B .2})$ are globally unstable. The proof of this results is based on developing the approach proposed in [13], [15] for studying the stability of solutions to hyperbolic equations.

\section{MAin RESUlT}

In what follows $W:=W_{0}^{1, p}(\Omega)$ denotes the Sobolev space, which is introduced as the completion $C_{0}^{\infty}(\Omega)$ by the norm:

$$
\|u\|_{1}=\left(\int_{\Omega}|\nabla u|^{p} d x\right)^{\frac{1}{p}} .
$$

The number $p^{*}$ denotes the critical Sobolev exponent defined as

$$
p^{*}= \begin{cases}+\infty, & n \leqslant p \\ \frac{p n}{n-p}, & n>p\end{cases}
$$

By $\langle\cdot, \cdot\rangle$ we denote the scalar product in the space $L^{2}(\Omega)$ and various duality in dual spaces. By $\lambda_{1}$ we denote the minimal eigenvalue of the operator $-\Delta_{p}$ subject to the Dirichlet boundary condition. As it is known,

$$
\lambda_{1}=\inf _{u \in W \backslash 0} \frac{\int_{\Omega}|\nabla u|^{p} d x}{\int_{\Omega}|u|^{p} d x} .
$$

Problem (B.1) has the variational formulation with the Euler-Lagrange functional of the following form:

$$
\Phi_{\lambda}(u)=\frac{1}{p} \int_{\Omega}|\nabla u|^{p} d x-\frac{\lambda}{p} \int_{\Omega}|u|^{p} d x+\frac{1}{\alpha} \int_{\Omega}|u|^{\alpha} d x, \quad u \in W .
$$

We consider weak solutions $u_{\lambda}$ to problem (B.1), that is, the critical points of functional (2):

$$
D_{u} \Phi_{\lambda}\left(u_{\lambda}\right)(\phi)=0 \text { for all } \phi \in W,
$$

where $D_{u} \Phi_{\lambda}\left(u_{\lambda}\right)$ is the Fréchet derivative. A weak solution $u_{\lambda}$ to problem $(\bar{B} .1)$ is called ground state (stationary ground state (B.2p) if the inequality $\Phi_{\lambda}\left(u_{\lambda}\right) \leqslant \Phi_{\lambda}\left(w_{\lambda}\right)$ holds for each other weak solution $w_{\lambda} \in W \backslash 0$ to problem (B.1). 
We study problem (B.1) by the Nehari manifold method. The corresponding minimization Nehari problem is introduced as

$$
\hat{\Phi}_{\lambda}=\inf \left\{\Phi_{\lambda}(u): u \in N_{\lambda}\right\}
$$

where

$$
N_{\lambda}=\left\{u \in W \backslash 0: \Phi_{\lambda}^{\prime}(u):=D_{u} \Phi_{\lambda}(u)(u)=0\right\}
$$

is the Nehari manifold. We observe that since each nontrivial solution to problem (B.1) belongs to $N_{\lambda}$ and $N_{\lambda}=\emptyset$ as $\lambda \leqslant \lambda_{1}$, then problem (B.1) has no solutions as $\lambda \leqslant \lambda_{1}$ except the trivial one $u \equiv 0$.

Our first main result is the following theorem.

Theorem 1. Let $1<\alpha<p<+\infty$, the boundary $\partial \Omega$ is a $C^{1, \gamma}$-manifold with some $\gamma \in(0,1]$. Then for all $\lambda>\lambda_{1}$

1. $\hat{\Phi}_{\lambda}>0$;

2. there exists a ground state $u_{\lambda}$ of problem B.1) and at that, $u_{\lambda} \geqslant 0$ in $\Omega$ and $u_{\lambda} \in C^{1, \beta}(\bar{\Omega})$ for some $\beta \in(0,1)$.

Remark 1. A similar result in the case $p=2$ was obtained in [5].

The functional

$$
E_{\lambda}(\xi, \zeta)=\frac{1}{2} \int_{\Omega}|\zeta|^{2} d x+\frac{1}{p} \int_{\Omega}|\nabla \xi|^{p} d x-\frac{\lambda}{p} \int_{\Omega}|\xi|^{p} d x+\frac{1}{\alpha} \int_{\Omega}|\xi|^{\alpha} d x, \quad \xi \in W, \zeta \in L^{2}(\Omega)
$$

is called the energy functional of problem (B.2).

Let $\left(v_{0}, v_{1}\right) \in W \times L^{2}(\Omega)$. Following work [13], we call $v(t):=v\left(t ; v_{0}, v_{1}\right)$ a weak solution to problem B.2 on $[0, T]$, where $T<+\infty$, if it satisfies the conditions:

$\left(1^{0}\right)$ the mapping $[0, T] \ni t \mapsto v(t) \in W$ is continuous in the weak topology of $W$;

$\left(2^{0}\right)$ there exists a mapping $[0, T] \ni t \mapsto v_{t}(t) \in L^{2}(\Omega)$ continuous in the weak topology of $L^{2}(\Omega)$ such that the identity

$$
\left.\langle v(t), \phi\rangle\right|_{t_{1}} ^{t_{2}}=\int_{t_{1}}^{t_{2}}\left\langle v_{s}(s), \phi\right\rangle d x
$$

holds true for all $t_{1}, t_{2}, 0 \leqslant t_{1}<t_{2} \leqslant T$ and all $\phi \in L^{2}(\Omega)$;

$\left(3^{0}\right)$ for each $w:[0, T] \rightarrow W$ satisfying properties $\left(1^{0}\right),\left(2^{0}\right)$ the identity

$$
\begin{aligned}
\left.\left\langle v_{t}(t), w(t)\right\rangle\right|_{t_{1}} ^{t_{2}}=\int_{t_{1}}^{t_{2}} & {\left[\left\langle v_{s}(s), w_{s}(s)\right\rangle-\left\langle|\nabla v(s)|^{p-2} \nabla v(s), \nabla w(s)\right\rangle\right.} \\
& \left.+\left\langle\lambda|v(s)|^{p-2} v(s)-|v(s)|^{\alpha-2} v(s), w(s)\right\rangle\right] d x
\end{aligned}
$$

holds true;

$\left(4^{0}\right)$ the inequality holds:

$$
E_{\lambda}\left(v(t), v_{t}(t)\right) \leqslant E_{\lambda}\left(v_{0}, v_{1}\right) \text { for all } t \in[0, T] .
$$

Let $T_{m}:=T_{m}\left(v_{0}, v_{1}\right) \in(0,+\infty]$ be the maximal value such that for all $T \in\left(0, T_{m}\right)$ there exists a weak solution $v\left(t ; v_{0}, v_{1}\right)$ to problem $(\mathrm{B} .2)$ on $[0, T]$. In the case $T_{m}=+\infty$ we shall say that the corresponding $v\left(t ; v_{0}, v_{1}\right)$ is a global solution of (B.2).

We note that Theorem 1 implies that problem (B.2) possesses a global solution. Indeed, the weak solution $u_{\lambda}$ to problem (B.1) is also a weak solution $u_{\lambda}\left(t ; u_{\lambda}, 0\right) \equiv u_{\lambda}$ to problem (B.2) on $[0,+\infty)$. 
However, since equation (B.2) involves the non-Lipschitz nonlinearity, the issue on whether problem (B.2) possesses a weak solution $v\left(t ; v_{0}, v_{1}\right)$ with some $T_{m}\left(v_{0}, v_{1}\right) \in(0,+\infty]$ for arbitrary $\left(v_{0}, v_{1}\right) \in W \times L^{2}(\Omega)$ is still open. In the present work we do not study this issue.

We shall say that a weak solution $v_{\lambda}\left(t ; v_{0}, v_{1}\right)$ of problem $(\mathrm{B} .2)$ is globally unstable if for each $\varepsilon>0$ there exist $\left(w_{0}, w_{1}\right) \in W \times L^{2}(\Omega):\left\|v_{0}-w_{0}\right\|_{1}<\varepsilon$ and $\left\|v_{1}-w_{1}\right\|_{L^{2}}<\varepsilon$ such that one of the following alternatives holds: 1) problem (B.2) has not weak solutions with the initial condition $\left.\left(w_{0}, w_{1}\right) ; 2\right)$ problem $(\mathrm{B} .2)$ has a weak solution $w_{\lambda}\left(t ; w_{0}, w_{1}\right)$ but $T_{m}\left(w_{0}, w_{1}\right)<+\infty$; $3)$ on $[0,+\infty)$, there exists a global solution $w_{\lambda}\left(t ; w_{0}, w_{1}\right)$ to problem (B.2) such that

$$
\int_{\Omega}\left|v_{\lambda}\left(t ; v_{0}, v_{1}\right)-w_{\lambda}\left(t ; w_{0}, w_{1}\right)\right|^{2} d x \rightarrow+\infty \quad \text { as } \quad t \rightarrow+\infty .
$$

The main result of the present is the following theorem.

Theorem 2. Let $1<\alpha<p<+\infty$, the boundary $\partial \Omega$ be a $C^{1, \gamma}$-manifold with some $\gamma \in(0,1]$. Then for all $\lambda>\lambda_{1}$ each ground state $u_{\lambda}$ of problem B.1) is globally unstable solution to hyperbolic problem (B.2).

Remark 2. In the present work we do not consider the existence of compactly supported in $\Omega$ ground states for problem (B.1). Nevertheless, Theorem 2 implies that if such solutions exist, they are globally unstable for (B.2).

\section{Existence of Ground state For PRoblem (B.1)}

We introduce the notations

$$
H_{\lambda}(u):=\int_{\Omega}|\nabla u|^{p} d x-\lambda \int_{\Omega}|u|^{p} d x, \quad F(u):=\int_{\Omega}|u|^{\alpha} d x .
$$

We consider Nehari functional

$$
\Phi_{\lambda}^{\prime}(u):=H_{\lambda}(u)+F(u) .
$$

It follows from (1) that $N_{\lambda} \neq \emptyset$ if and only if $\lambda>\lambda_{1}$. We observe that $\Phi_{\lambda}(u)>0$ for all $u \in N_{\lambda}$. Indeed, if $u \in N_{\lambda}$, then $H_{\lambda}(u)=-F(u)$ that by $1<\alpha<p$ yields:

$$
\Phi_{\lambda}(u)=\frac{p-\alpha}{p \alpha} F(u)>0 .
$$

We consider minimization problem (4). We note that for each $u \in N_{\lambda}$

$$
D_{u u} \Phi_{\lambda}(u)(u, u)=(\alpha-p) \int_{\Omega}|u|^{\alpha} d x<0 .
$$

As it is known (see [7], [11]), condition (12) is sufficient for each solution $u$ to problem (4) is a critical point of the functional $\Phi_{\lambda}(u)$.

We consider the fibering functional 14

$$
J_{u}(r)=\Phi_{\lambda}(r u), \quad r>0 .
$$

Let $H_{\lambda}(u)<0$. Then there exists the unique root $r^{*}=r^{*}(u)>0$ of the equation

$$
J_{u}^{\prime}(r)=r^{p-1} H_{\lambda}(u)+r^{\alpha-1} F(u)=0 .
$$

Indeed, taking into consideration $H_{\lambda}(u)<0$, we find

$$
r^{*}(u):=\left(\frac{F(u)}{-H_{\lambda}(u)}\right)^{\frac{1}{p-\alpha}} .
$$

As one can see easily, $r^{*}(u)$ is the global maximum for $J_{u}(r)$. This implies that problem (4) is equivalent to the following one:

$$
\hat{\Phi}_{\lambda}=\inf \left\{\Phi_{\lambda}\left(r^{*}(v) v\right):\|v\|_{1}=1, H_{\lambda}(v)<0\right\}
$$


where

$$
\Phi_{\lambda}\left(r^{*}(v) v\right)=\frac{(p-\alpha)}{p \alpha} \frac{F(v)^{\frac{p}{p-\alpha}}}{\left(-H_{\lambda}(v)\right)^{\frac{\alpha}{p-\alpha}}} .
$$

Proof of Theorem 1. We consider the minimizing sequence $\left(v_{n}\right)$ of problem (16), that is,

$$
\lim _{n \rightarrow+\infty} \Phi_{\lambda}\left(r^{*}\left(v_{n}\right) v_{n}\right)=\hat{\Phi}_{\lambda} .
$$

We note that $\left\|v_{n}\right\|_{1}=1$ and hence, by Banach-Alaoglu theorem and by Sobolev theorem there exists a subsequence redenoted by $\left(v_{n}\right)$ such that $v_{n} \rightarrow w$ weakly in $W$ and $v_{n} \rightarrow w$ strongly in $L^{\gamma}(\Omega)$ as $1 \leqslant \gamma<p^{*}$.

Let us show that $w \neq 0$. We assume the opposite: let $w=0$, then $\int_{\Omega}\left|v_{n}\right|^{p} d x \rightarrow 0$ and in this case

$$
\int_{\Omega}\left|\nabla v_{n}\right|^{p} d x-\lambda \int_{\Omega}\left|v_{n}\right|^{p} d x=1-\lambda \int_{\Omega}\left|v_{n}\right|^{p} d x \geqslant 0
$$

for sufficiently large $n$. But this contradicts the condition that $H_{\lambda}\left(v_{n}\right)<0$ for $n=1,2, \ldots$ Therefore, $w \neq 0$. By 11 this implies $\hat{\Phi}_{\lambda}>0$.

We consider

$$
u_{n}=r^{*}\left(v_{n}\right) v_{n}
$$

We note that $r^{*}\left(v_{n}\right)$ is bounded. Indeed, it follows from (15) and the said above that $F\left(v_{n}\right)$ is bounded and $H_{\lambda}\left(v_{n}\right)$ does not tend to zero. By 15 we hence have

$$
0<C_{1}<r^{*}\left(v_{n}\right)<C_{2}<+\infty,
$$

where $C_{1}, C_{2}$ are independent of $n$. Therefore, there exists a limiting point $u_{\lambda}$ such that some subsequence redenoted by $\left(u_{n}\right)$ satisfies the convergence $u_{n} \rightarrow u_{\lambda}$ weakly in $W$ and $u_{n} \rightarrow u_{\lambda}$ strongly in $L^{\gamma}(\Omega)$ as $1 \leqslant \gamma<p^{*}$. By the weak lower semi-continuity of the norm $\|\cdot\|_{1}$ this implies:

$$
\begin{aligned}
& \left\|u_{\lambda}\right\|_{1} \leqslant \liminf _{n \rightarrow+\infty}\left\|u_{n}\right\|_{1}=d, \\
& \Phi_{\lambda}\left(u_{\lambda}\right) \leqslant \liminf _{n \rightarrow+\infty} \Phi_{\lambda}\left(u_{n}\right)=\hat{\Phi}_{\lambda} \quad \text { and } \quad \Phi_{\lambda}^{\prime}\left(u_{\lambda}\right) \leqslant \liminf _{n \rightarrow+\infty} \Phi_{\lambda}^{\prime}\left(u_{n}\right)=0 .
\end{aligned}
$$

Thus, if $\left\|u_{\lambda}\right\|_{1}=d$, then $\Phi_{\lambda}^{\prime}\left(u_{\lambda}\right)=0, \Phi_{\lambda}\left(u_{\lambda}\right)=\hat{\Phi}_{\lambda}$ and $u_{\lambda}$ is a non-zero minimizer for (4).

Assume that $\left\|u_{\lambda}\right\|_{1}<d$. Then $\Phi_{\lambda}^{\prime}\left(u_{\lambda}\right)<0$ and $\Phi_{\lambda}\left(u_{\lambda}\right)<\hat{\Phi}_{\lambda}$. Let us show that this is impossible. Indeed, in this case there exists $\bar{t} \in(0,1)$ such that $\Phi_{\lambda}^{\prime}\left(\bar{t} u_{\lambda}\right)=0$. We observe that since $\lim _{n \rightarrow+\infty} F\left(u_{n}\right) \rightarrow F\left(u_{\lambda}\right)$, we have

$$
\frac{1}{p} \lim _{n \rightarrow+\infty} H_{\lambda}\left(u_{n}\right)=\hat{\Phi}_{\lambda}-\frac{1}{\alpha} F\left(u_{\lambda}\right) \quad \text { and } \quad \lim _{n \rightarrow+\infty} H_{\lambda}\left(u_{n}\right)=-F\left(u_{\lambda}\right)
$$

This yields

$$
\begin{aligned}
\Phi_{\lambda}\left(\bar{t} u_{\lambda}\right) & =\frac{\bar{t}^{p}}{p} H_{\lambda}\left(u_{\lambda}\right)+\frac{\bar{t}^{\alpha}}{\alpha} F\left(u_{\lambda}\right)<\frac{\bar{t}^{p}}{p} \liminf _{n \rightarrow+\infty} H_{\lambda}\left(u_{n}\right)+\frac{\bar{t}^{\alpha}}{\alpha} F\left(u_{\lambda}\right) \\
& =\hat{\Phi}_{\lambda}-\frac{1}{\alpha} F\left(u_{\lambda}\right)-\frac{\bar{t}^{p}-1}{p} F\left(u_{\lambda}\right)+\frac{\bar{t}^{\alpha}}{\alpha} F\left(u_{\lambda}\right)=\hat{\Phi}_{\lambda}+\left(\frac{1-\bar{t}^{p}}{p}+\frac{\bar{t}^{\alpha}-1}{\alpha}\right) F\left(u_{\lambda}\right) .
\end{aligned}
$$

We denote

$$
I(t)=\left(\frac{1-\bar{t}^{p}}{p}+\frac{\bar{t}^{\alpha}-1}{\alpha}\right) F\left(u_{\lambda}\right) .
$$

The values of this functional on the boundary are

$$
I(0)=\left(\frac{1}{p}-\frac{1}{\alpha}\right) F\left(u_{\lambda}\right)<0, \quad I(1)=0 .
$$


At that,

$$
I^{\prime}(t)=\left(-t^{p-1}+t^{\alpha-1}\right) F\left(u_{\lambda}\right)>0 \text { for all } t \in(0,1) .
$$

Therefore, $I(t)<0$ for $0<t \leqslant 1$ and $\Phi_{\lambda}\left(\bar{t} u_{\lambda}\right)<\hat{\Phi}_{\lambda}$. However, $\Phi_{\lambda}^{\prime}\left(\bar{t} u_{\lambda}\right)=0$, that is, $\bar{t} u_{\lambda} \in N_{\lambda}$. We have obtained the contradiction with the definition of $\hat{\Phi}_{\lambda}$. Therefore, $u_{\lambda}$ is indeed a minimizer for (4).

We note that $\Phi_{\lambda}(u)=\Phi_{\lambda}(|u|)$ for all $u \in W$ and if $u \in N_{\lambda}$, then $|u| \in N_{\lambda}$. Therefore, $\left|u_{\lambda}\right|$ is also a solution to problem (4), that is, we can suppose that $u_{\lambda} \geqslant 0$ on $\Omega$. We note that by the assumption, the boundary $\partial \Omega$ is a $C^{1, \gamma}$-manifold for some $\gamma \in(0,1]$. Applying now the standard regularity theory for solutions to quasilinear boundary value problems [6], [12], [17], we therefore obtain that $u_{\lambda} \in C^{1, \beta}(\bar{\Omega})$ for some $\beta \in(0,1)$. The proof is complete.

\section{MAIN LEMMA}

The next lemma will be employed below for studying stability of solution to (B.2).

Lemma 1. Let $\left(u_{n}\right)$ be a sequence in $W \backslash 0$ such that $\Phi_{\lambda}^{\prime}\left(u_{n}\right) \leqslant 0$ and $\Phi_{\lambda}^{\prime}\left(u_{n}\right) \rightarrow 0$. Then the inequality

holds true.

$$
\liminf _{n \rightarrow+\infty} \Phi_{\lambda}\left(u_{n}\right) \geqslant \hat{\Phi}_{\lambda}
$$

Proof. Assume that we are given a sequence $\left(u_{n}\right) \subset W \backslash 0$ such that $\Phi_{\lambda}^{\prime}\left(u_{n}\right) \leqslant 0$ and $\Phi_{\lambda}^{\prime}\left(u_{n}\right) \rightarrow 0$. We write this sequence as $u_{n}=r_{n} v_{n}$, where $r_{n}=\left\|u_{n}\right\|_{1}$ and $\left\|v_{n}\right\|_{1}=1$. Since $\left(v_{n}\right)$ is bounded in $W$, then by Banach-Alaoglu theorem and by Sobolev theorem, without loss of generality we can suppose that $v_{n} \rightarrow \bar{v}$ converges strongly in $L^{\gamma}(\Omega)$ as $1 \leqslant \gamma<p^{*}$ and $v_{n} \rightarrow \bar{v}$ weakly in $W$ for some $\bar{v} \in W$.

Let us prove that $\bar{v}$ is non-zero; we shall argue by contradiction. Indeed, let $v_{n} \rightarrow 0$ in $L^{p}(\Omega)$. Then $H_{\lambda}\left(v_{n}\right) \rightarrow 1$ and $F\left(v_{n}\right) \rightarrow 0$. However, this implies that

$$
1 \longleftarrow H_{\lambda}\left(v_{n}\right) \leqslant H_{\lambda}\left(v_{n}\right)+r_{n}^{\alpha-p} F\left(v_{n}\right)=\frac{1}{r_{n}^{p}} \Phi_{\lambda}^{\prime}\left(u_{n}\right) \leqslant 0,
$$

the contradiction.

Let us prove that $r_{n}$ does not tend to zero. Assume the opposite: $r_{n} \rightarrow 0$. Then

$$
H_{\lambda}\left(v_{n}\right)+r_{n}^{\alpha-p} F\left(v_{n}\right) \rightarrow+\infty
$$

since $F_{\alpha}\left(v_{n}\right) \rightarrow F(\bar{v}) \neq 0$. We again obtained the contradiction.

Assume that $r_{n}$ tends to infinity. Then $F\left(u_{n}\right)=r_{n}^{\alpha} F\left(v_{n}\right) \rightarrow+\infty$ as $n \rightarrow+\infty$ and since $H_{\lambda}\left(u_{n}\right)+F\left(u_{n}\right) \rightarrow 0$, then $-H_{\lambda}\left(u_{n}\right) \rightarrow+\infty$ as $n \rightarrow+\infty$. Hence,

$$
\Phi_{\lambda}\left(u_{n}\right)=\left(\frac{\alpha-p}{p \alpha}\right) H_{\lambda}\left(u_{n}\right)+\frac{1}{\alpha} \Phi_{\lambda}^{\prime}\left(u_{n}\right) \rightarrow+\infty .
$$

Therefore, inequality 23 is true.

Now we consider the case, when $\left(r_{n}\right)$ is bounded and therefore, $\left(u_{n}\right)$ is bounded. Then as above, without loss of generality, we can assume that there exists a limit $w$ such that $u_{n} \rightarrow w$ strongly in $L^{\gamma}(\Omega)$ as $1 \leqslant \gamma<p^{*}$ and $u_{n} \rightarrow w$ weakly in $W$. Arguing as above, we show that $w \neq 0$ and $\Phi_{\lambda}^{\prime}(w) \leqslant 0$. Therefore, if $\lim _{n \rightarrow+\infty}\left\|u_{n}\right\|_{1}=\|w\|_{1}$, then $\Phi_{\lambda}^{\prime}(w)=0$ and we obtain the desired identity

$$
\lim _{n \rightarrow+\infty} \Phi_{\lambda}\left(u_{n}\right)=\Phi_{\lambda}(w) \geqslant \hat{\Phi}_{\lambda}
$$

On other hand, the situation $\Phi_{\lambda}^{\prime}(w)<0$ is impossible.

Indeed, assume that $\Phi_{\lambda}^{\prime}(w)<0$. Then there exists $\bar{t} \in(0,1)$ such that $\Phi_{\lambda}^{\prime}(\bar{t} w)=0$. Arguing as in the proof of Theorem 1, we obtain the following inequality:

$$
\Phi_{\lambda}(\bar{t} w) \leqslant \liminf _{n \rightarrow+\infty} \Phi_{\lambda}\left(u_{n}\right)+I(\bar{t}),
$$


where $I(t)$ is the function defined in (21). As we have shown above, $I(\bar{t})<0$. This is why, if $\liminf _{n \rightarrow+\infty} \Phi_{\lambda}\left(u_{n}\right)<\hat{\Phi}_{\lambda}$, then $\Phi_{\lambda}(\bar{t} w)<\hat{\Phi}_{\lambda}$. However, $\Phi_{\lambda}^{\prime}(\bar{t} w)=0$, that is, $\bar{t} w \in N_{\lambda}$. We have obtained a contradiction with the definition of $\hat{\Phi}_{\lambda}$.

\section{Proof of global instability of problem (B.2)}

In this section we prove Theorem 2 .

We introduce the set

$$
\Theta=\left\{(\xi, \psi) \in(W \backslash 0) \times L^{2}(\Omega): E_{\lambda}(\xi, \psi)<\hat{\Phi}_{\lambda}, \Phi_{\lambda}^{\prime}(\xi)<0\right\} .
$$

We note that the ground state $u_{\lambda}$ lies on the boundary of the set $\bar{\Theta}$ since $E_{\lambda}\left(u_{\lambda}, 0\right)=\hat{\Phi}_{\lambda}$, $\Phi_{\lambda}^{\prime}\left(u_{\lambda}\right)=0$.

Assume that there exists a weak solution $v(t)$ to problem $(\overline{B .2})$ defined on some maximal interval $\left[0, T_{m}\right), T_{m} \leqslant+\infty$. We observe that $\left(2^{0}\right)$ implies that the mapping $v(t)$ is weakly absolutely continuous from $[0, T]$ into $L^{2}(\Omega)$ for all $T \in\left(0, T_{m}\right)$. Indeed, since $v_{t}(t)$ is a weakly continuous mapping from $[0, T]$ into $L^{2}(\Omega)$, by $(6)$ we get the inequality

$$
\left|\left\langle v\left(t_{2}\right), \phi\right\rangle-\left\langle v\left(t_{1}\right), \phi\right\rangle\right| \leqslant \int_{t_{1}}^{t_{2}}\left|\left\langle v_{s}(s), \phi\right\rangle\right| d x \leqslant \max _{s \in[0 ; T]}\left|\left\langle v_{s}(s), \phi\right\rangle\right|\left(t_{2}-t_{1}\right) \quad \text { for all } \phi \in L^{2}(\Omega),
$$

which means that $\langle v(t), \phi\rangle$ satisfies the Lipschitz condition on $[0, T]$ and therefore, it is absolutely continuous on $[0, T]$ for all $\phi \in L^{2}(\Omega)$.

Lemma 2. Let

$$
L(t)=\int_{\Omega} v^{2}(x, t) d x
$$

where $v(x, t)$ is a weak solution to $\overline{B .2})$. Then for almost all $t \in(0, T)$ there exists the derivative $\dot{L}(t)$. Moreover, as $T \in\left(0, T_{m}\right)$, almost everywhere on $(0, T)$ there exists $\ddot{L}(t)$ and the identity

$$
\ddot{L}(t)=2 \int_{\Omega}\left(\left|v_{t}(t)\right|^{2}-|\nabla v(t)|^{p}+\lambda|v(t)|^{p}-|v(t)|^{\alpha}\right) d x \quad \text { a.e. on }(0, T)
$$

holds true.

Proof. We introduce the function $P(t, s)=\langle v(t), v(s)\rangle$. Since $v(t)$ is a weakly absolutely continuous function from $[0, T]$ into $L^{2}(\Omega)$, the function $P(t, s)$ is differentiable almost everywhere in $t \in(0, T)$ and $s \in(0, T)$. Taking into consideration that $v_{t}$ is weakly continuous from $[0, T]$ into $L^{2}(\Omega)$, by (6) we get that all partial derivatives $\frac{\partial}{\partial t} P(t, s)$ and $\frac{\partial}{\partial s} P(t, s)$ are continuous. By differentiability property of functions of many variables this implies that the function $P(t, s)$ is differentiable at the point $(t, t)$. Thus, there exists $\dot{L}(t)$ and

$$
\dot{L}(t)=\left.\left(\frac{\partial}{\partial t} P(t, s)+\frac{\partial}{\partial s} P(t, s)\right)\right|_{s=t}=2\left\langle v_{t}(t), v(t)\right\rangle .
$$

We note that (7) with $\phi=v(t)$ gives

$$
\left.\left\langle v_{t}(t), v(t)\right\rangle\right|_{t_{1}} ^{t_{2}}=\int_{t_{1}}^{t_{2}} \int_{\Omega}\left(\left|v_{s}(s)\right|^{2}-|\nabla v(s)|^{p}+\lambda|v(s)|^{p}-|v(s)|^{\alpha}\right) d x d x .
$$

Then

$$
\dot{L}\left(t_{2}\right)-\dot{L}\left(t_{1}\right)=2 \int_{t_{1}}^{t_{2}} \int_{\Omega}\left(\left|v_{s}(s)\right|^{2}-|\nabla v(s)|^{p}+\lambda|v(s)|^{p}-|v(s)|^{\alpha}\right) d x d x .
$$

By the absolute continuity of the Lebesgue integral this implies that $\dot{L}(t)$ is an absolutely continuous function that implies the existence of the derivative $\ddot{L}(t)$ almost everywhere on $(0, T)$ and the validity of $(30)$. 
Lemma 3. Let $v(t)$ be a global solution of (B.2) such that $\left(v(0), v_{t}(0)\right) \in \Theta$. Then $\left(v(t), v_{t}(t)\right) \in \Theta$ for all $t \in[0,+\infty)$.

Proof. Let $v(t)$ be a global solution to (B.2) subject to the initial conditions $v(0)=v_{0}, v_{t}(0)=v_{1}$ such that $\left(v_{0}, v_{1}\right) \in \Theta$. Then by (8),

$$
\Phi_{\lambda}(v(t)) \leqslant E\left(v(t), v_{t}(t)\right) \leqslant E\left(v_{0}, v_{1}\right)<\hat{\Phi}_{\lambda} \text { for all } t \in[0,+\infty) .
$$

Assume the opposite, that $\left(v(t), v_{t}(t)\right)$ leaves the domain $\Theta$. By $(32)$, this is possible only if there exists $t_{0}$ such that $\Phi_{\lambda}^{\prime}\left(v\left(t_{0}\right)\right) \geqslant 0$. We denote by $t_{1}$ the smallest time when $\Phi_{\lambda}^{\prime}\left(v\left(t_{1}\right)\right) \geqslant 0$. Then $\Phi_{\lambda}^{\prime}(v(t))<0$ as $0 \leqslant t<t_{1}$ and $\Phi_{\lambda}^{\prime}\left(v\left(t_{1}\right)\right) \geqslant 0$. The weak lower semi-continuity of the norm $\|\cdot\|_{1}$ implies

$$
0 \leqslant \Phi_{\lambda}^{\prime}\left(v\left(t_{1}\right)\right) \leqslant \liminf _{t \uparrow t_{1}} \Phi_{\lambda}^{\prime}(v(t)) \leqslant 0
$$

Therefore, the inequality $\Phi_{\lambda}^{\prime}\left(v\left(t_{1}\right)\right)>0$ is impossible. Assume that $\Phi_{\lambda}^{\prime}\left(v\left(t_{1}\right)\right)=0$. Then $v\left(t_{1}\right) \in N_{\lambda}$ and therefore, $\Phi_{\lambda}\left(v\left(t_{1}\right)\right) \geqslant \hat{\Phi}_{\lambda}$; but this contradicts 32. The proof is complete.

Lemma 4. If $\left(v_{0}, v_{1}\right) \in \Theta$ and $v(t)$ is a global solution to $\left(B\right.$.2), then $\int_{\Omega}|v(t)|^{2} d x \rightarrow+\infty$ as $t \rightarrow+\infty$.

Proof. We consider $L(t)=\int_{\Omega}|v(t)|^{2} d x$. Then by Lemma 2 we have $\dot{L}(t)=2\left\langle v_{t}(t), v(t)\right\rangle$ and

$$
\ddot{L}(t)=2\left(\int_{\Omega}\left|v_{t}(t)\right|^{2} d x-\int_{\Omega}|\nabla v(t)|^{p} d x+\lambda \int_{\Omega}|v(t)|^{p} d x-\int_{\Omega}|v(t)|^{\alpha} d x\right)
$$

almost everywhere on $(0, T)$. By Lemma 3

$$
-\Phi_{\lambda}^{\prime}(v(t))=-\int_{\Omega}|\nabla v(t)|^{p} d x+\lambda \int_{\Omega}|v(t)|^{p} d x-\int_{\Omega}|v(t)|^{\alpha} d x \geqslant 0 \quad \text { for all } \quad t>0 .
$$

This implies that $\ddot{L}(t) \geqslant 0$ a.e on $(0, T)$.

Let us show that there exists $t_{0}>0$ such that $\dot{L}\left(t_{0}\right)>0$. We assume the opposite, that is, $\dot{L}(t) \leqslant 0$ for all $t>0$. Since $L(t)>0$ and $L(t)$ is convex, then $L(t)$ should tend for a finite constant as $t \rightarrow+\infty$. Therefore, $L(t) \rightarrow A, \dot{L}(t) \rightarrow 0, \ddot{L}(t) \rightarrow 0$ as $t \rightarrow+\infty$ for some $A \in[0,+\infty)$. Then by (33) and (34) we obtain

$$
\lim _{t \rightarrow+\infty} \int_{\Omega}\left|v_{t}(t)\right|^{2} d x=0 .
$$

Hence, in view of the fact it follows from 8 that

$$
\frac{1}{2} \int_{\Omega}\left|v_{t}(t)\right|^{2} d x+\frac{1}{p} \int_{\Omega}|\nabla v(t)|^{p} d x-\frac{\lambda}{p} \int_{\Omega}|v(t)|^{p} d x+\frac{1}{\alpha} \int_{\Omega}|v(t)|^{\alpha} d x \leqslant E\left(v_{0}, v_{1}\right),
$$

we get the estimate

$$
\liminf _{t \rightarrow+\infty} \Phi_{\lambda}(v(t)) \leqslant E\left(v_{0}, v_{1}\right) .
$$

On the other hand, since $\ddot{L}(t) \rightarrow 0$ as $t \rightarrow+\infty$, then 33 and 35 implies that

$$
\Phi_{\lambda}^{\prime}(v(t))=\int_{\Omega}|\nabla v(t)|^{p} d x-\lambda \int_{\Omega}|v(t)|^{p} d x+\int_{\Omega}|v(t)|^{\alpha} d x \rightarrow 0
$$

as $t \rightarrow+\infty$. Hence, taking into consideration (34), by Lemma 1 we get

$$
\liminf _{t \rightarrow+\infty} \Phi_{\lambda}(v(t)) \geqslant \hat{\Phi}_{\lambda}>E\left(v_{0}, v_{1}\right) .
$$

This is opposite to 36 . Thus, indeed, there exists $t_{0}>0$ such that $\dot{L}\left(t_{0}\right)>0$.

Since $\ddot{L}(t) \geqslant 0$, we can write

$$
\int_{t_{0}}^{t} \ddot{L}(s) d x=\dot{L}(t)-\dot{L}\left(t_{0}\right) \geqslant 0 .
$$


Then

$$
\int_{t_{0}}^{t}\left(\dot{L}(s)-\dot{L}\left(t_{0}\right)\right) d x=L(t)-L\left(t_{0}\right)-\dot{L}\left(t_{0}\right)\left(t-t_{0}\right) \geqslant 0 .
$$

Since $\dot{L}\left(t_{0}\right)>0$, we conclude that

$$
L(t)=\int_{\Omega}|v(t)|^{2} d x \geqslant \dot{L}\left(t_{0}\right)\left(t-t_{0}\right)+L\left(t_{0}\right) \rightarrow+\infty .
$$

Completion of the proof of Theorem 2. Let $u_{\lambda}$ be a ground state of (B.1) and $\varepsilon>0$. We consider $r>1$ satisfying the inequality

$$
|r-1|<\frac{\varepsilon}{\left\|u_{\lambda}\right\|_{1}} .
$$

Then $\left\|u_{\lambda}-r u_{\lambda}\right\|_{1}<\varepsilon$. We shall prove the theorem if we show that for each global solution $v\left(t ; v_{0}, v_{1}\right)$ of problem (B.2) with the initial conditions $v_{0}=r u_{\lambda}, v_{1}=0$ (assuming the existence of such solution) the identity

$$
\lim _{t \rightarrow+\infty} \int_{\Omega}\left|u_{\lambda}-v\left(t ; v_{0}, v_{1}\right)\right|^{2} d x=+\infty
$$

holds. Let us prove this.

As $r>1$, the inequalities

$$
E_{\lambda}\left(r u_{\lambda}, 0\right)<\hat{\Phi}_{\lambda}, \Phi_{\lambda}^{\prime}\left(r u_{\lambda}\right)<0
$$

hold. They mean that $\left(r u_{\lambda}, 0\right) \in \Theta$. Then by Lemma 4 we have

$$
\int_{\Omega}\left|v\left(t ; v_{0}, v_{1}\right)\right|^{2} d x \rightarrow+\infty \quad \text { as } \quad t \rightarrow+\infty
$$

which implies $(39)$.

\section{BIBLIOGRAPHY}

1. H. Brezis. Solutions of variational inequalities with compact support // Uspekhi Matem. Nauk. 29:2(176), 103-108 (1974). [Russ. Math. Surv. 29:2, 103-108 (1974).]

2. J.I. Díaz. Nonlinear partial differential equations and free boundaries. Pitman Research Notes in Mathematics Series. 106. Pitman Publ. Ltd., London (1985).

3. J.I. Díaz On the Haïm Brezis pioneering contributions on the location of free boundaries // in "Proceedings of the Fifth European Conference on Elliptic and Parabolic Problems; A special tribute to the work of Haïm Brezis". Birkhäuser, Basel. 217-234 (2005).

4. J.I. Díaz, J. Hernández, Y. Ilyasov. Flat solutions of some non-Lipschitz autonomous semilinear equations may be stable for $N \geqslant 3 / /$ Chinese Ann. Math. Ser. B. 38:1, 345-378 (2017).

5. J.I. Díaz, J. Hernández, Y. Ilyasov On the existence of positive solutions and solutions with compact support for a spectral nonlinear elliptic problem with strong absorption // Nonl. Anal.: Theory, Methods and Appl. 119, 484-500 (2015).

6. E. DiBenedetto. $C^{1+\alpha}$ local regularity of weak solutions of degenerate elliptic equations // Nonl. Anal.: Theory, Methods and Appl. 7:8, 827-850 (1983).

7. Y.S. Il'yasov. Nonlocal investigations of bifurcations of solutions of nonlinear elliptic equations // Izv. Math. 66:6, 1103-1130 (2002).

8. Y.S. Ilyasov, Y. Egorov. Höpf maximum principle violation for elliptic equations with nonLipschitz nonlinearity // Nonl. Anal.: Theory, Methods and Appl. 72:7-8, 3346-3355 (2010).

9. Y. Il'yasov. A duality principle corresponding to the parabolic equations // Physica D: Nonl. Phenom. 237:5, 692-698 (2008).

10. Y.S. Il'yasov. On critical exponent for an elliptic equation with non-Lipschitz nonlinearity // Discr. Contin. Dynam. Syst. Suppl. 698-706 (2011). 
11. Y.S. Il'yasov. On extreme values of Nehari manifold method via nonlinear Rayleigh's Quotient // Topolog. Meth. Nonl. Anal. 49:2, 683-714 (2017).

12. G.M. Lieberman. Boundary regularity for solutions of degenerate elliptic equations // Nonl. Anal.: Theory, Methods and Appl. 12:11, 1203-1219 (1988).

13. L.E. Payne and D.H. Sattinger. Saddle points and instability of nonlinear hyperbolic equations // Israel J. Math. 22:3, 273-303 (1975).

14. S.I. Pohozaev. On the method of fibering a solution in nonlinear boundary value problems // Proc. Stekl. Ins. Math. 192, 146-163 (1990).

15. D.H. Sattinger. On global solution of nonlinear hyperbolic equations // Arch. Ration. Mech. Anal. 30:2, 148-172 (1968).

16. J. Serrin and H. Zou. Symmetry of ground states of quasilinear elliptic equations // Arch. Ration. Mech. Anal. 148:4, 265-290 (1999).

17. P. Tolksdorf. Regularity for a more general class of quasilinear elliptic equations // J. Diff. Equat. 51:1, 126-150 (1984).

Yavdat Shavkatovich Il'yasov, Institute of Mathematics, Ufa Scientific Center, RAS, Chernyshevsky str. 112, 450077, Ufa, Russia E-mail: Ilyasov02@gmail.com

Emil' Eduardovich Kholodnov, Institute of Mathematics, Ufa Scientific Center, RAS, Chernyshevsky str. 112, 450077, Ufa, Russia

E-mail: emil.kholod@gmail.com 